\title{
Substrate Stiffness Affected the Inflammatory Response of SMCs
}

\author{
Xiuli Mao' ${ }^{*}$, Lixuan Mao², Hong Zhang1, Yiling Tan¹, Huali Wang1 \\ ${ }^{1}$ School of Biomedical Engineering, Shanghai Jiao Tong University, Shanghai, China \\ ${ }^{2}$ School of Engineering, Computing and Mathematics, Plymouth University, Plymouth, UK \\ Email: *xiuli.mao@sjtu.edu.cn
}

How to cite this paper: Mao, X.L., Mao, L.X., Zhang, H., Tan, Y.L. and Wang, H.L. (2021) Substrate Stiffness Affected the Inflammatory Response of SMCs. Journal of Biosciences and Medicines, 9, 44-54. https://doi.org/10.4236/jbm.2021.93006

Received: February 8, 2021

Accepted: March 19, 2021

Published: March 22, 2021

Copyright $\odot 2021$ by author(s) and Scientific Research Publishing Inc. This work is licensed under the Creative Commons Attribution International License (CC BY 4.0).

http://creativecommons.org/licenses/by/4.0/

\begin{abstract}
The pathogenesis of atherosclerosis is accompanied by chronic inflammation with changes in the stiffness of the coronary artery wall. Being the main component of the vascular media, the smooth muscle cells (SMCs) are crucial to maintain blood vessel function. SMCs are mechano-sensitive, which can rapidly adapt to the fluctuations in the microenvironment of the blood vessel, including the subtle changes of the vascular stiffness. However, how substrate stiffness influences the phenotype and inflammatory response of SMCs is not well understood. In this study, we investigated the effects of substrate stiffness on SMCs phenotype, inflammatory gene expression and the nuclear factorkappa $\mathrm{B}(\mathrm{NF} \kappa \mathrm{B})$ signaling pathway of vascular SMCs. From $1 \mathrm{kPa}$ to $100 \mathrm{kPa}$, the SMCs cytoskeleton became more and more organized with the increase of the substrate stiffness, representing by the uniformed distribution of the stress fibers. SMCs cultured on both soft $(1 \mathrm{kPa})$ and hard $(100 \mathrm{kPa})$ substrate increased the expression of macrophage marker CD68 molecule (CD68) and Galectin 3 (LGALS3) and the inflammatory gene Interleukin-6 (IL-6) and Interleukin-1 $\beta$ (IL-1 $\beta$ ) than those on $40 \mathrm{kPa}$ substrate. Moreover, the protein expression level of phosphorylated nuclear factor kappa B inhibitor ( $\mathrm{p}-\mathrm{I} \kappa \mathrm{B})$ was higher on either soft $(1 \mathrm{kPa})$ or hard $(100 \mathrm{kPa})$ substrate. In consistent, the dephosphorylated $\mathrm{I} \kappa \mathrm{B}$ showed a higher expression level on the substrate stiffness of $40 \mathrm{kPa}$. These results suggested that substrate stiffness played an important role in SMCs cell morphology, phenotype and inflammatory response by affecting $\mathrm{NF} \kappa \mathrm{B}$ signaling pathway.
\end{abstract}

\section{Keywords}

Vascular Smooth Muscle Cells, Substrate Stiffness, Inflammatory Response, $\mathrm{NF} \kappa \mathrm{B}$ 


\section{Introduction}

Atherosclerosis, a major cause of heart attack and stroke, is associated with chronic inflammation in the coronary arteries [1]. Innate immune cells such as macrophages are the main cause of vascular inflammation. Studies have shown that in the early stage of atherosclerosis, monocytes adhere to endothelial cells, migrate to the intima, and then mature into macrophages [2]. Macrophages play an important role in atherosclerotic formation, including the clearance of lipoproteins, cytokines and reactive oxygen, which may lead to plaque rupture and thrombosis [3]. More recently, Bennett and Sinha et al. found that smooth muscle cells in atherosclerotic plaques can change their phenotype to macrophage-likecells, and then affect the inflammation of the blood vessels [4]. It has been reported that more than $50 \%$ of $\mathrm{CD}^{+} 8^{+}$foam cells are SMCs-derived in atherosclerotic lesion [5].

Moreover, the vascular environment could change with the development of atherosclerosis. The deposition of lipoproteins in the blood vessels will soften the blood vessels. Subsequently, excessive lipid leads to extracellular matrix, fibrous tissue hyperplasia and calcareous deposition, which gradually increases vascular stiffness, and finally affects the vascular environmental homeostasis [6] [7]. In the physiological state, the stiffness of aorta is about $40 \mathrm{kPa}$. In contrast, the pathological condition could cause stiffness to decrease to as low as $1 \mathrm{kPa}$ and increase to even $100 \mathrm{kPa}$ [8] [9]. Substrate stiffness can affect the chemotaxis and adhesion of monocytes by regulating the microRNA expression level of endothelial cells [10]. Meanwhile, SMCs can also sense and respond to substrate stiffness changes in the vascular wall, causing phenotypic switching. Tian et al. found that as substrate stiffness increases, SMCs transform from a synthetic phenotype to a contractile phenotype, and substrate stiffness regulates SMCs matrix remodeling through TGF- $\beta$ signal pathway. [11]. Hence, it is necessary to explore the effects of substrate stiffness on the SMCs' immune response in the atherosclerosis.

Nuclear transcription factor $\kappa \mathrm{B}(\mathrm{NF} \kappa \mathrm{B})$, the central transcriptional control point in vascular inflammation, is a key regulator in atherosclerosis. Furthermore, NF $\kappa$ B plays a vital role in a complex system that allows cells to adapt and respond to environmental changes. The activation of $\mathrm{NF} \kappa \mathrm{B}$ depends on phosphorylation-induced degradation of the $\mathrm{NF} \kappa \mathrm{B}$ protein inhibitor $\mathrm{I} \kappa \mathrm{B}$ [12]. It has been reported that the activation of $\mathrm{NF} \kappa \mathrm{B}$ can be induced by both physically (ultraviolet exposure) and physiologically (ischemia) factors [13] [14]. There is an evidence that $\mathrm{NF} \kappa \mathrm{B}$ was activated by low extracellular $\mathrm{Mg}^{2+}$ in cerebrovascular smooth muscle cells [15]. Macrophages also have been shown to induce the expression of associated inflammatory cytokines (IL-6, TNF- $\alpha$, IL-1) through the activation of $\mathrm{NF} \kappa \mathrm{B}[16]$.

Therefore, the effects of substrate stiffness on the SMCs, including cells morphology, phenotype and immune response were studied. Furthermore, whether $\mathrm{NF} \kappa \mathrm{B}$ signaling pathway was activated in response of SMCs to the substrate 
stiffness was also investigated.

\section{Materials and Methods}

\subsection{Cell Culture and Treatment}

The human aortic smooth muscle cells (SMCs) were grown in SMCs medium (Scien Cell, USA), supplemented with $2 \%$ fetal bovine serum, $1 \%$ penicillin/streptomycin, and $1 \%$ smooth muscle cell growth supplement. SMCs were cultured in a $37^{\circ} \mathrm{C}$ incubator (Corning, USA) with the supplement of $5 \% \mathrm{CO}_{2}$.

The SMCs were cultured on polyacrylamide hydrogels with different stiffness for 24 hours before proceeding to the experiments.

\subsection{Preparation of Substrates with Different Stiffness}

Poly-acrylamide hydrogel substrates with different stiffness were prepared as described previously by adjusting the ratio of $40 \%$ acrylamide (w/v) (Sangon Biotech, China) to $2 \%$ bis-acrylamide (w/v) (Sangon Biotech, China) [11]. Tetramethylethylenediamine (Klamar, China) and 10\% ammonium persulfate solution (Sinopharm, China) were added to the mixture for cross-linking. Before polymerization, the mixture was added to a glass slide and a silicified cover glass was put on the gel droplets to form a leveled surface. On completion of the polymerization, the glass slide was removed and the cover glass with the substrates were treated with sulfo-SANPAH (Thermo Fisher, USA) and coated with 1 $\mathrm{mg} / \mathrm{mL}$ Collagen Type I (Corning, USA) to facilitate cell adhesion. The stiffness of the substrates were measured by an atomic force microscope. The substrates were sterilized by UV and 75\% alcohol for 30 minutes before cell culture.

\subsection{Real-Time Quantitative PCR (RT-qPCR)}

Total RNA was extracted from SMCs using the RNA simple Total RNA Extraction kit (TIANGEN, China). Reverse transcription was performed using the Fastking RT kit with gDNase (TIANGEN, China). For RT-qPCR, the SuperRealPreMix Plus kit (SYBR Green) (TIANGEN, China) was used. The primer pairs sequences were listed in Table 1. The mRNA expression of the targetgene was expressed as fold of change to GAPDH control.

Table 1. RT-qPCR primer sequences.

\begin{tabular}{ccc}
\hline $\begin{array}{c}\text { Primer } \\
\text { name }\end{array}$ & Forward primer (5'-3’) & Reverse primer (5'-3’) \\
\hline GAPDH & GGGAAGGTGAAGGTCGGAGT & GGGGTCATTGATGGCAACA \\
MYH11 & GCTCAGAAAGTTTGCCACCTC & CATCCGCCCAACCTTGATA \\
IL-6 & ATGAGGAGACTTGCCTGGTG & GTGAGGAACAAGCCAGAGCT \\
IL-1 $\beta$ & TCTTCATTGCTCAAGTGTCTG & TGCCACTGTAATAAGCCATC \\
LGALS3 & CATGCTGATAACAATTCTGGG & GGTTAAAGTGGAAGGCAACAT \\
\hline
\end{tabular}




\subsection{Western Blotting}

Total SMCs lysates were extracted in the plus RIPA lysis buffer (Beyotime, China) with protease and phosphatase inhibitor. BCA Protein Assay Kit (Thermo Scientific, USA) was used to quantify the protein concentration. Equal amount of the total cell lysates was subject to the SDS-PAGE, and transferred to PVDF membrane. After 10\% BSA blocking, primary antibodies of CD68 (Abcam, UK), p-I $\kappa$ B (Cell Signaling Technology, USA), I $\kappa$ B (Cell Signaling Technology, USA), GAPDH (Cell Signaling Technology, USA) and $\alpha$-Tubulin (Cell Signaling Technology, USA) were incubated overnight at $4^{\circ} \mathrm{C}$, respectively. HRP-conjugated secondary antibodies (Cell Signaling Technology, USA) were incubated for 1hour at room temperature. The bands were visualized by Immobilon western chemiluminescent HRP substrate (Merck Millipore, Germany). The protein expression was normalized to $\alpha$-Tubulin (Cell Signaling Technology, USA) in the respective samples.

\subsection{Immunofluorescence Staining and Image Processing}

SMCs were fixed in $4 \%(\mathrm{w} / \mathrm{v})$ PFA for 10 minutes. Then, F-actin was stained with phalloidin conjugated with iFluorTM488 (AAT Bioquest, USA) for $90 \mathrm{mi}-$ nutes. Finally, cell nucleus was stained with DAPI for 5 minutes. Images were captured under a laser scanning confocal microscope (Leica TCS SP5 II, Germany).

For image processing, as described in Z. Püspöki et al. [17], the local orientation properties of images are computed according to the Orientation J software and were then visualized as color images with the orientation being typically encoded in the color (hue).

\subsection{Statistical Analysis}

For all the experiments, at least 3 independent trials were performed. Unless otherwise indicated, data were expressed as mean \pm SEM. Pairwise comparisons were made using a Student t-test. Comparison of three or more groups was performed using One-way ANOVA. A value of ${ }^{\star} p<0.05$ was considered statistically significant. Data were analyzed by GraphPad Prism 6.0 software.

\section{Results}

\subsection{Substrate Stiffness Affected SMCs Morphology.}

SMCs were grown on different stiffness for 24 hours and subjected to phalloidin staining for F-actin filaments representing the stress fibers in the cell. The morphology of SMCs exhibited significant difference and the average F-acting fluorescence intensity increased with the increase of substrate stiffness (Figure 1(A) and Figure 1(B)). The F-actin stress fiber orientation colormaps (Figure 1(C)) were analyzed by Orientation J software. The color maps of actin stress fibers showed that SMCs on the $1 \mathrm{kPa}$ substrate exhibited mixed colors, whereas the color maps for SMCs on 40 and $100 \mathrm{kPa}$ substrate exhibited similar colors. In 


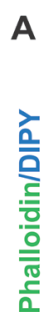

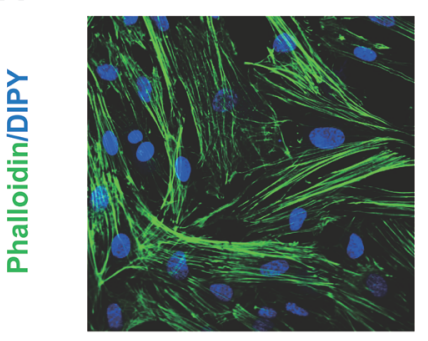

$40 \mathrm{kPa}$

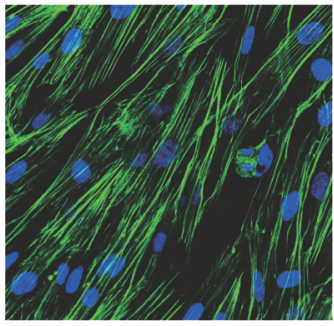

$100 \mathrm{kPa}$

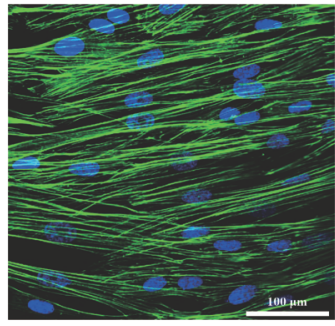

B

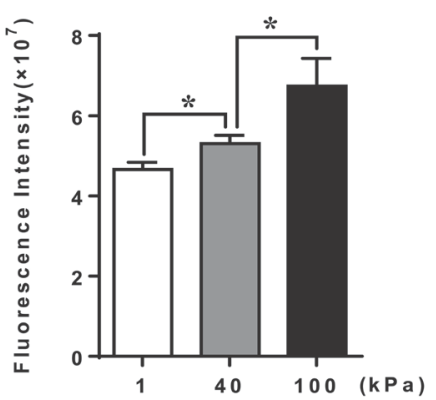

C

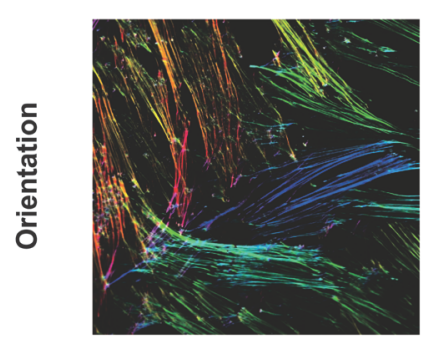

$40 \mathrm{kPa}$

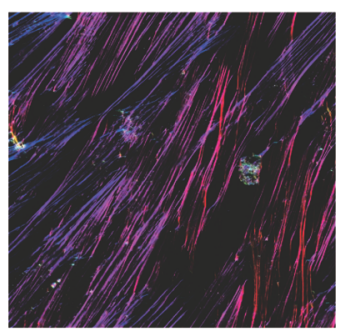

$100 \mathrm{kPa}$

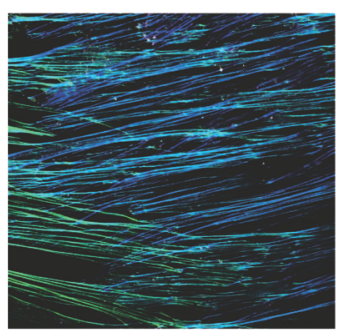

color-scale

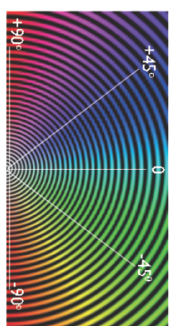

D
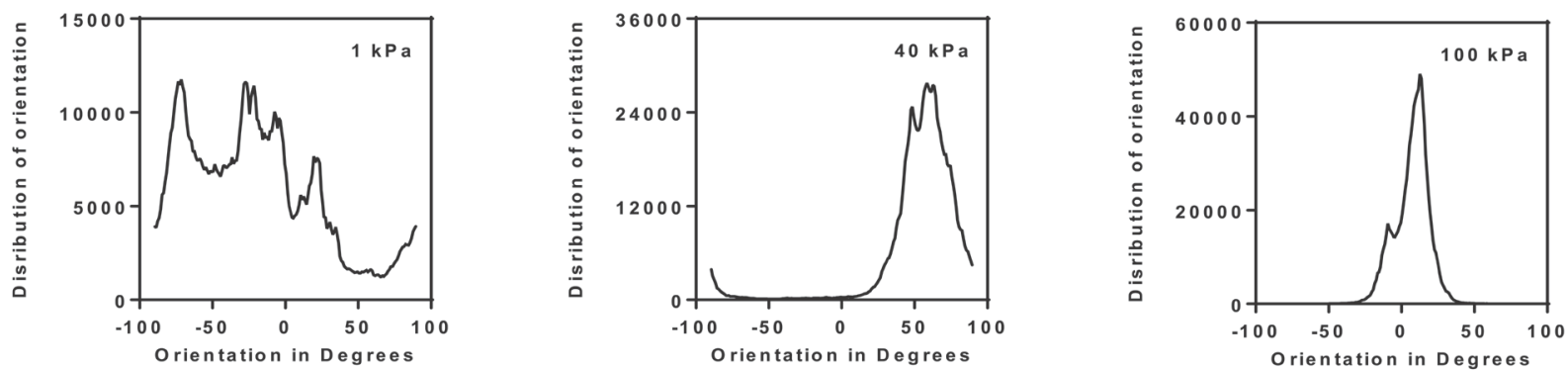

Figure 1. Substrate stiffness affected SMCs morphology. SMCs were seeded on different substrate stiffness (1 kPa, $40 \mathrm{kPa}, 100$ $\mathrm{kPa}$ ) for 24 hours. (A): Immunofluorescence staining of F-actin (green), DAPI staining of nucleus (blue) (scale bar, $100 \mu \mathrm{m})$. (B): The amount of F-acting was quantified by the fluorescence intensity. (C): The color maps for SMCs F-acting orientation on different substrate stiffness. (D): Quantitation of the distribution of SMCs F-acting orientation on different substrate stiffness. *indicates $p<0.05$.

addition, the distribution of stress fibers orientation on different substrate stiffness were counted. The results showed that, with the increasing of substrate stiffness, the distribution of stress fibers orientation is more condensed, indicating a more organized overall SMCs cytoskeleton (Figure 1(D)). Taken together, these results suggested that substrate stiffness affected SMCs morphology and cytoskeleton organization.

\subsection{Substrate Stiffness Regulated SMCs Phenotype}

SMC is an undifferentiated cell type with significant phenotypic plasticity. It has 
been shown that increased extracellular substrate stiffness drives the development of arterial pathology and changes the phenotype of vascular cells. We analyzed the effect of substrate stiffness on the phenotype of SMCs. With the treatment of different stiffness, the gene expression of SMC contractile maker MYH11 and the macrophage marker LGALS3 and CD68 were examined. The results showed the expression of SMC contractile maker MYH11 was increased (Figure 2(A)) with the increase of substrate stiffness. And SMCs expressed higher LGALS3 on both soft (1 kPa) and hard (100 kPa) substrates (Figure 2(B)) than on $40 \mathrm{kPa}$ substrate. Consistently, the Western blotting results showed that the expression level of CD68 was the lowest on $40 \mathrm{kPa}$ substrate (Figure 2(C), Figure 2(D)).

\subsection{Substrate Stiffness Affected SMCs Inflammatory Response}

The acquisition of macrophage phenotypes is associated with inflammatory responses. To further explore the inflammatory response of SMCs on different

A

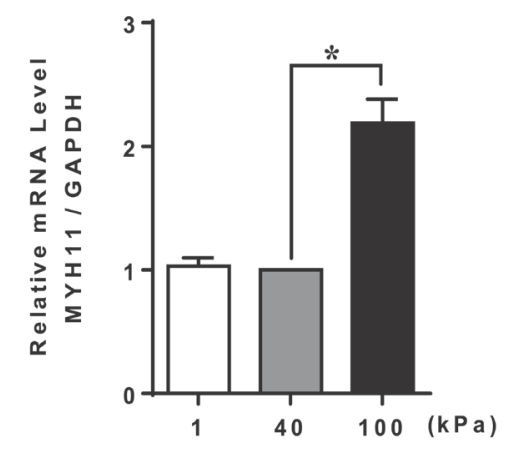

C

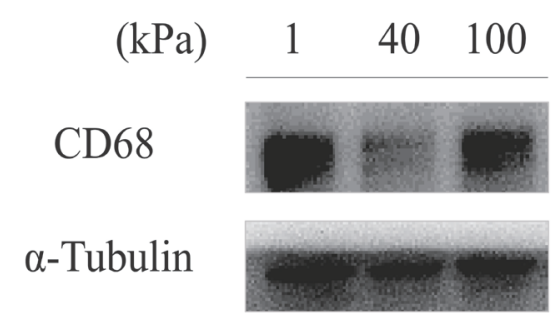

B

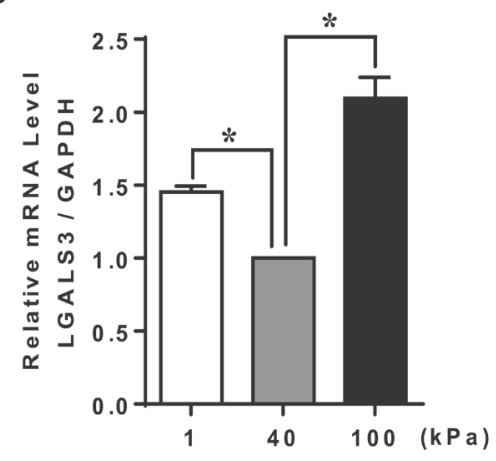

D

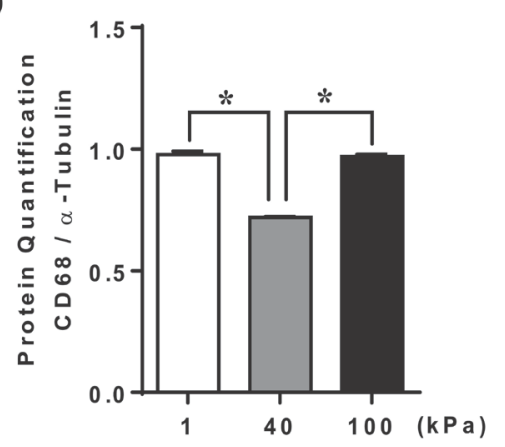

Figure 2. Substrate stiffness affected SMCs phenotype. SMCs were seeded on different substrate stiffness ( $1 \mathrm{kPa}, 40 \mathrm{kPa}, 100 \mathrm{kPa}$ ) for 24 hours. (A): SMC marker MYH11 gene expression level was measured by RT-qPCR. (B): Macrophage marker LGALS3 gene expression level was measured by RT-qPCR. GAPDH was used as the internal control for normalization $(\mathrm{n}=3)$. (C): Macrophage marker CD68 protein expression was analyzed by Western blotting analysis. The expression of $\alpha$-Tubulin was used as the internal control. (D): Quantitation of the CD68 protein expression was calculated as the ratio of target protein to $\alpha$-Tubulin $(\mathrm{n}=3)$. *indicates $p<0.05$. 
substrate stiffness, the expression of inflammation-related genes was examined in this study. RT-qPCR results showed that, same as the LGALS3 and CD68 the gene expression of IL- 6 and IL- $1 \beta$ were higher on both soft $(1 \mathrm{kPa})$ and hard $(100 \mathrm{kPa})$ substrates (Figure 3(A), Figure 3(B)). SMCs on the $40 \mathrm{kPa}$ substrate shown a relatively low level of gene expression, suggesting lower inflammation on $40 \mathrm{kPa}$ substrate.

\subsection{Substrate Stiffness Affected NF $\kappa$ B Signaling Pathway of SMCs}

In vivo and in vitro experiments studies have shown that $\mathrm{NF} \kappa \mathrm{B}$ is activated in an inflammatory environment. Here, the Western blotting analysis used to detect the activity of $\mathrm{NF} \kappa \mathrm{B}$ signaling related molecules. The results showed that, compared with the $40 \mathrm{kPa}$, the protein expression of $\mathrm{p}-\mathrm{I} \kappa \mathrm{B}$ was higher at $1 \mathrm{kPa}$ and $100 \mathrm{kPa}$. On the contrary, the protein level of $\mathrm{I} \kappa \mathrm{B}$ was lower at $40 \mathrm{kPa}$ than that on $1 \mathrm{kPa}$ and $100 \mathrm{kPa}$ substrates (Figure 4). These results suggested that $\mathrm{NF} \kappa \mathrm{B}$ signaling pathway was involved in the inflammatory response of SMCs.

A

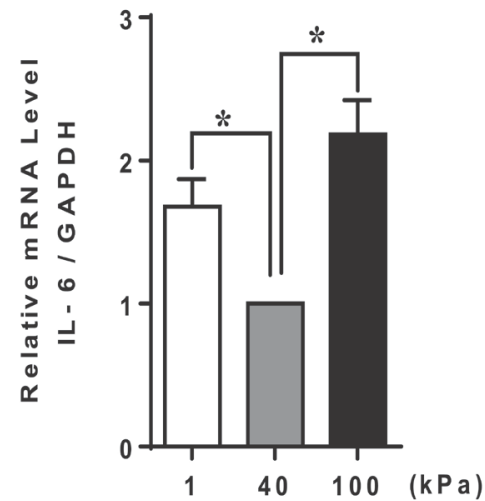

B

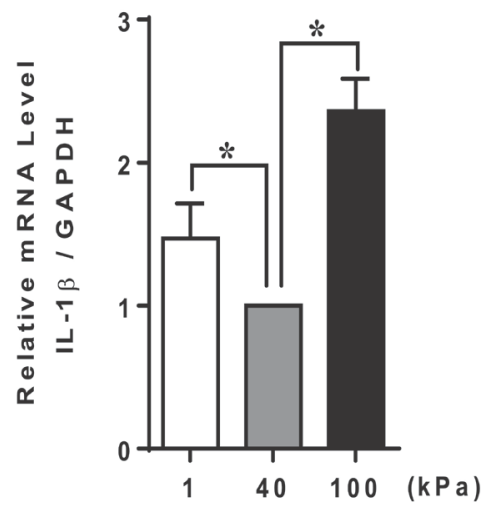

Figure 3. Substrate stiffness affected SMCs inflammatory response. SMCs were seeded on different substrate stiffness $(1 \mathrm{kPa}, 40 \mathrm{kPa}, 100 \mathrm{kPa})$ for 24 hours. (A): IL-6 expression level was measured by RT-qPCR. (B): IL- $1 \beta$ expression level was measured by RT-qPCR. GAPDH was used as the internal control for normalization $(\mathrm{n}=3)$. ${ }^{\star}$ indicates $p<0.05$.

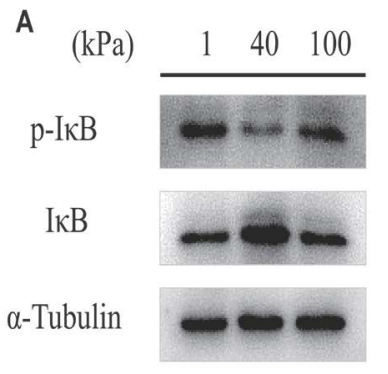

B

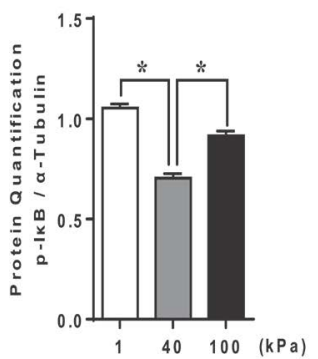

C

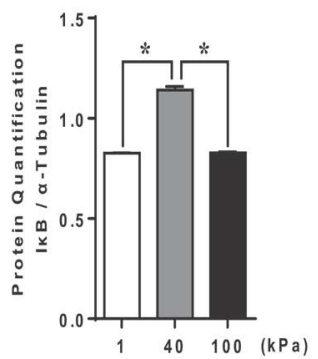

Figure 4. Substrate stiffness affected $\mathrm{NF} \kappa \mathrm{B}$ signaling pathway of SMCs. SMCs were seeded on different substrate stiffness $(1 \mathrm{kPa}, 40 \mathrm{kPa}, 100 \mathrm{kPa})$ for 24 hours. (A): I $\kappa \mathrm{B}$ and $\mathrm{p}-\mathrm{I} \kappa \mathrm{B}$ protein expression was analyzed by Western blotting analysis. The expression of $\alpha$-Tubulin was used as the internal control. (B), (C): Quantitation of the protein expression was calculated as the ratio of target protein to $\alpha$-Tubulin $(\mathrm{n}=3)$. ${ }^{\star}$ indicates $p<0.05$. 


\section{Discussion and Conclusion}

In the process of atherosclerosis, the vascular microenvironment, such as high lipid, reactive oxygen species, substrate stiffness, will change, and then affect the development of lesions. Studies have shown that, compared with normal arteries, the aortic elastic modulus of rabbits fed with high cholesterol gradually decreased within 1 - 8 weeks. Afterwards, with the development of the lesion, the elastic modulus of the aorta gradually increases until calcification [18]. Meanwhile, studies have shown that vascular remodeling may contribute to medial and intimal calcification and increase vascular stiffness [19]. Therefore, it is meaningful to pay attention to the effect of substrate stiffness on the function and structure of vascular cells.

Smooth muscle cells are the main component of the vascular media. Their main function is contract, which can regulate the tension of blood vessels and maintain blood vessel function [20]. In addition, smooth muscle cells exhibit significant phenotypic plasticity and they can rapidly adapt to fluctuating environmental signals [21]. More and more evidences show that SMCs have undergone significant cytoskeletal remodeling during migration and phenotypic transformation [11] [22]. It is reported that vasoconstrictors and vasodilators can induce changes in vascular smooth muscle cytoskeletal remodeling [23]. Our results also showed that substrate stiffness affected SMCs cytoskeleton remodels and phenotypic transformation. The SMCs cytoskeleton were progressively disrupted following the decreasing substrate stiffness (Figure 1). In addition, in vitro studies, the substrate stiffness showed a significant effect on the autophagy of smooth muscle cells. With the increment of substrate stiffness, the level of autophagy of smooth muscle cells increased and the phenotypic transition of smooth muscle cells was activated [24]. Our results showed that as the stiffness of the substrate changes, SMCs showed a phenotypic switch to foam cells. SMCs expressed higher LGALS3 and CD68 on both soft $(1 \mathrm{kPa})$ and hard $(100 \mathrm{kPa})$ substrates maybe because $1 \mathrm{kPa}$ and $100 \mathrm{kPa}$ substrate stiffness are the pathological stiffness of atherosclerosis which more easily to induce SMCs phenotypic transformation.

It is well known that atherosclerosis is intimately linked with inflammation. Studies have shown that the inflammation-related genes including IL-6, IL-1 $\beta$, MCP-1, VCAM-1, etc. showed a significantly up-regulated in atherosclerotic susceptible areas [24] [25]. The inflammatory response of SMCs on variational substrate stiffnesses was investigated in this study. Compared with the middle substrate stiffness $(40 \mathrm{kPa})$, the expression of inflammatory factors IL- 6 and IL- $1 \beta$ are higher on the softer $(1 \mathrm{kPa})$ and harder $(100 \mathrm{kPa})$ substrate stiffness, which means that a moderate substrate stiffness is beneficial to cellular function. In addition, $\mathrm{NF} \kappa \mathrm{B}$ is a key regulator of inflammation and atherosclerosis [26]. The activation of $\mathrm{NF} \kappa \mathrm{B}$ can be initiated under cytokine signaling or environmental stress [27]. Typical pathways for $\mathrm{NF} \kappa \mathrm{B}$ activation include the rapid degradation of $\mathrm{I} \kappa \mathrm{B}$ by proteasomes, and the release of $\mathrm{NF} \kappa \mathrm{B}$ subunits. Then, the 
$\mathrm{NF} \kappa \mathrm{B}$ subunits translocate to the nucleus and bind to homologous DNA motifs of target genes to promote transcription [28]. Autieri and Yue et al. found that activation of $\mathrm{NF} \kappa \mathrm{B}$ signaling pathway could inhibit the adhesion and proliferation of human vascular smooth muscle cells and can prevent the formation of new intima in rat carotid arteries [29]. Interestingly, our results showed that SMCs had higher expression of $\mathrm{p}-\mathrm{I} \kappa \mathrm{B}$ on softer $(1 \mathrm{kPa})$ and harder $(100 \mathrm{kPa})$ substrate stiffness to promote inflammation. It is suggested that a reasonable substrate stiffness can suppress SMCs inflammation by regulating $\mathrm{NF} \kappa \mathrm{B}$ signal pathway.

In summary, this study found that substrate stiffness regulated SMCs morphology and cytoskeleton organization. Furthermore, substrate stiffness also affected the phenotypic transformation and inflammatory response of SMCs by activating $\mathrm{NF} \kappa \mathrm{B}$ signaling pathway. This is complementary to our current understanding of the mechanism and pathogenesis of atherosclerosis.

\section{Conflicts of Interest}

The authors report no commercial or proprietary interest in any product or concept discussed in this article.

\section{References}

[1] Libby, P. (2012) Inflammation in Atherosclerosis. Arteriosclerosis, Thrombosis, and Vascular Biology, 32, 2045-2051. https://doi.org/10.1161/ATVBAHA.108.179705

[2] Moore, K.J. and Tabas, I. (2011) Macrophages in the Pathogenesis of Atherosclerosis. Cell, 145, 341-355. https://doi.org/10.1016/j.cell.2011.04.005

[3] Moore, K.J., Sheedy, F.J. and Fisher, E.A. (2013) Macrophages in Atherosclerosis: A Dynamic Balance. Nature Reviews Immunology, 13, 709-721. https://doi.org/10.1038/nri3520

[4] Bennett, M.R., Sinha, S. and Owens, G.K. (2016) Vascular Smooth Muscle Cells in Atherosclerosis. Circulation Research, 118, 692-702. https://doi.org/10.1161/CIRCRESAHA.115.306361

[5] Allahverdian, S., Chehroudi, A.C., McManus, B.M., Abraham, T. and Francis, G.A. (2014) Contribution of Intimal Smooth Muscle Cells to Cholesterol Accumulation and Macrophage-Like Cells in Human Atherosclerosis. Circulation, 129, 1551-1559. https://doi.org/10.1161/CIRCULATIONAHA.113.005015

[6] Dubland, J.A. and Francis, G.A. (2016) So Much Cholesterol: The Unrecognized Importance of Smooth Muscle Cells in Atherosclerotic Foam Cell Formation. Current Opinion in Lipidology, 27, 155-161. https://doi.org/10.1097/MOL.0000000000000279

[7] Lyle, A.N. and Raaz, U. (2018) Killing Me Un-Softly: Causes and Mechanisms of Arterial Stiffness. Arteriosclerosis, Thrombosis, and Vascular Biology, 37, e1-e11. https://doi.org/10.1161/ATVBAHA.116.308563

[8] Tracqui, P., Broisat, A., Toczek, J., Mesnier, N., Ohayon, J. and Riou, L. (2011) Mapping Elasticity Moduli of Atherosclerotic Plaque in Situ via Atomic Force Microscopy. Journal of Structural Biology, 174, 115-123. https://doi.org/10.1016/j.jsb.2011.01.010

[9] Matsumoto, T., Abe, H., Ohashi, T., Kato, Y. and Sato, M. (2002) Local Elastic 
Modulus of Atherosclerotic Lesions of Rabbit Thoracic Aortas Measured by Pipette Aspiration Method. Physiological Measurement, 23, 635-648. https://doi.org/10.1088/0967-3334/23/4/304

[10] Chen, W.C., Tian, B.X., Liang, J.Q., Yu, S.Y., Zhou, Y. and Li, S. (2019) Matrix Stiffness Regulates the Interactions between Endothelial Cells and Monocytes. Biomaterials, 221, Article ID: 119362.

https://doi.org/10.1016/j.biomaterials.2019.119362

[11] Tian, B.X., Ding, X.L., Song, Y., Chen, W.C., Liang, J.Q., Yang, L., Fan, Y.B., Li, S. and Zhou, Y. (2019) Matrix Stiffness Regulates SMC Functions via TGF- $\beta$ Signaling Pathway. Biomaterials, 221, Article ID: 119407.

https://doi.org/10.1016/j.biomaterials.2019.119407

[12] Baeuerle, P.A. and Henkel, T. (1994) Function and Activation of NF- $\kappa$ B in the Immune System. Annual Review of Immunology, 12, 141-179.

https://doi.org/10.1146/annurev.iy.12.040194.001041

[13] Verma, A., Kushwaha, H.N., Srivastava, A.K., Srivastava, S., Jamal, N., Srivastava, K. and Ray, R.S. (2017) Piperine Attenuates UV-R Induced Cell Damage in Human Keratinocytes via NF- $\kappa \mathrm{B}, \mathrm{Bax} / \mathrm{Bcl}-2$ Pathway: An Application for Photoprotection. Journal of Photochemistry and Photobiology B Biology, 172, 139-148. https://doi.org/10.1016/j.jphotobiol.2017.05.018

[14] Xia, J.B., Liu, G.H., Chen, Z.Y., Mao, C.Z., Zhou, D.C., Wu, H.Y., Park, K.S., Zhao, H., Kim, S.K., Cai, D.Q. and Qi, X.F. (2016) Hypoxia/Ischemia Promotes CXCL10 Expression in Cardiac Microvascular Endothelial Cells by NF- $\kappa$ B Activation. Cytokine, 81, 63-70. https://doi.org/10.1016/j.cyto.2016.02.007

[15] Altura, B.M., Kostellow, A.B., Zhang, A.M., Li, W.Y., Morrill, G.A., Gupta, R.K. and Altura, B.T. (2003) Expression of NFB and Proto-Oncogenes c-fos and c-jun Are Induced by Low Magnesium in Aortic and Cerebral Vascular Smooth Muscle Cells: Possible Link to Hypertension, Atherogenesis, and Stroke. American Journal of Hypertension, 16, 701-707. https://doi.org/10.1016/S0895-7061(03)00987-7

[16] Liddle, D.M., Monk, J.M., Hutchinson, A.L., Ma, D.W.L. and Robinson, L.E. (2019) $\mathrm{CD}^{+} \mathrm{T}$ Cell/Adipocyte Inflammatory Cross Talk and Ensuing M1 Macrophage Polarization Are Reduced by Fish-Oil-Derived n-3 Polyunsaturated Fatty Acids, in Part by a TNF-Alpha-Dependent Mechanism. Journal of Nutritional Biochemistry, 76, Article ID: 108243. https://doi.org/10.1016/j.jnutbio.2019.108243

[17] Püspöki, Z., Storath, M., Sage, D. and Unser, M. (2016) Transforms and Operators for Directional Bioimage Analysis: A Survey. Advances in Anatomy, Embryology and Cell Biology, 219, 69-93. https://doi.org/10.1007/978-3-319-28549-8_3

[18] Rattazzi, M., Bertacco, E., Puato, M., Faggin, E. and Pauletto, P. (2012) Hypertension and Vascular Calcification: A Vicious Cycle? Journal of Hypertension, 30, 1885-1893. https://doi.org/10.1097/HJH.0b013e328356c257

[19] Chang, W.G. and Niklason, L.E. (2017) A Short Discourse on Vascular Tissue Engineering. NPJ Regenerative Medicine, 2, 7. https://doi.org/10.1038/s41536-017-0011-6

[20] Gomez, D. and Owens, G.K. (2012) Smooth Muscle Cell Phenotypic Switching in Atherosclerosis. Cardiovascular Research, 95, 156-164. https://doi.org/10.1093/cvr/cvs115

[21] Kappert, K., Blaschke, F., Meehan, W.P., Kawano, H., Grill, M., Fleck, E, Hsueh, W.A., Law, R.E. and Graf, K. (2001) Integrins Alphavbeta3 and Alphavbeta5 Mediate VSMC Migration and Are Elevated During Neointima Formation in the Rat Aorta. Basic Research in Cardiology, 96, 42-49. 
https://doi.org/10.1007/s003950170076

[22] Hong, Z.K., Reeves, K.J., Sun, Z., Li, Z.H., Brown, N.J. and Meininger, G.A. (2015) Vascular Smooth Muscle Cell Stiffness and Adhesion to Collagen I Modified by Vasoactive Agonists. PLoS ONE, 10, e0119533.

https://doi.org/10.1371/journal.pone.0119533

[23] Hu, M., Jia, F., Huang, W.P., Li, X., Hu, D.F., Wang, J., Ren, K.F., Fu, G.S., Wang, Y.-B. and Ji, J. (2020) Substrate Stiffness Differentially Impacts Autophagy of Endothelial Cells and Smooth Muscle Cells. Bioactive Materials, 6, 1413-1422. https://doi.org/10.1016/j.bioactmat.2020.10.013

[24] Fang, Y., Shi, C.Z., Manduchi, E., Civelek, M. and Davies, P.F. (2010) Microrna-10a Regulation of Proinflammatory Phenotype in Athero-Susceptible Endothelium in Vivo and in Vitro. Proceedings of the National Academy of Sciences of the United States of America, 107, 13450-13455. https://doi.org/10.1073/pnas.1002120107

[25] Liang, J.Q., Gu, S.Y., Mao, X.L., Tan, Y.L., Wang, H.L., Li, S. and Zhou, Y. (2020) Endothelial Cell Morphology Regulates Inflammatory Cells through microRNA Transferred by Extracellular Vesicles. Frontiers in Bioengineering and Biotechnology, 19, 369. https://doi.org/10.3389/fbioe.2020.00369

[26] Kempe, S., Kestler, H., Lasar, A. and Wirth, T. (2005) NF- $\kappa$ B Controls the Global Pro-Inflammatory Response in Endothelial Cells: Evidence for the Regulation of a Proatherogenic Program. Nucleic Acids Research, 33, 5308-5319. https://doi.org/10.1093/nar/gki836

[27] Denk, A., Goebeler, M., Schmid, S., Berberich, I., Ritz, O., Lindemann, D., Ludwig, S. and Wirth, T. (2001) Activation of NF-kappa B via the Ikappa B Kinase Complex Is Both Essential and Sufficient for Proinflammatory Gene Expression in Primary Endothelial Cells. Journal of Biological Chemistry, 276, 28451-28458. https://doi.org/10.1074/jbc.M102698200

[28] Oeckinghaus, A. and Ghosh, S. (2009) The NF- $\kappa$ B Family of Transcription Factors and Its Regulation. Cold Spring Harbor Symposia on Quantitative Biology, 1, a000034. https://doi.org/10.1101/cshperspect.a000034

[29] Autieri, M.V., Yue, T.L., Ferstein, G.Z. and Ohlstein, E. (1995) Antisense Oligonucleotides to the p65 Subunit of NF- $\kappa$ B Inhibit Human Vascular Smooth Muscle Cell Adherence and Proliferation and Prevent Neointima Formation in Rat Carotid Arteries. Biochemical and Biophysical Research Communications, 213, 827-836. https://doi.org/10.1006/bbrc.1995.2204 